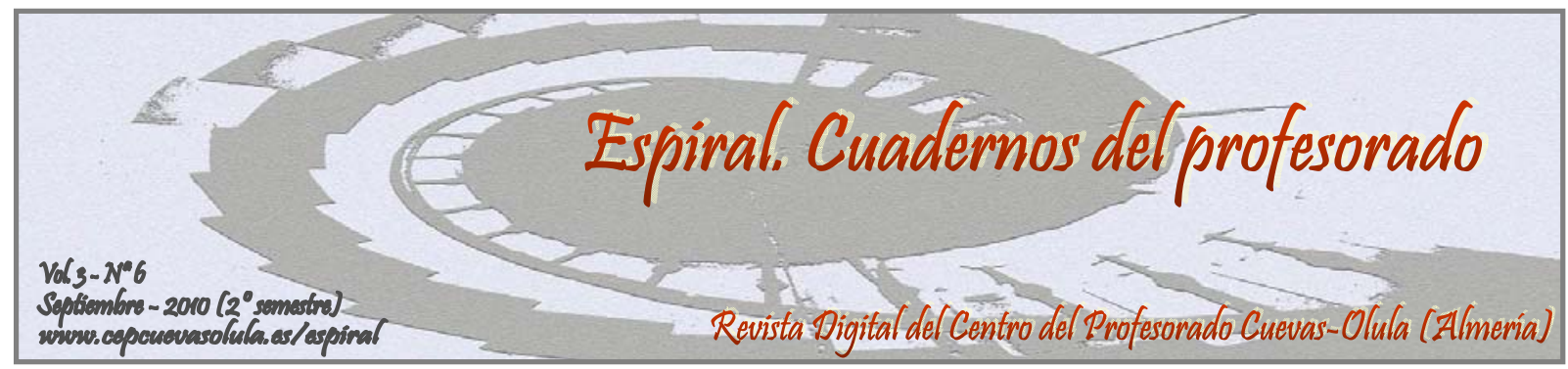

\title{
LA TUTORIA COMO VEHÍCULO PARA EL FOMENTO DE LAS COMPETENCIAS TRANSVERSALES EN LOS NUEVOS GRADOS DE INGENIERÍA AGRONÓMICA
}

\section{THE TUTORING AS A VEHICLE FOR THE PROMOTION OF COMPETENCES IN THE NEW GRADE CROSS AGRICULTURAL ENGINEERING}

\author{
Carlos San-Antonio-Gómez ${ }^{(1)}$ y Francisco Manzano-Agugliaro ${ }^{(2)}$
}

\author{
${ }^{(1)}$ E. T. Superior de Ingenieros Agrónomos, Universidad Politécnica de Madrid, España \\ ${ }^{(2)}$ Escuela Politécnica Superior, Universidad de Almería, España
}

\begin{abstract}
RESUMEN: Los nuevos títulos de grado incorporan las competencias transversales, dedicadas a la formación en valores humanos del alumnado. Además estos nuevos títulos tienen la particularidad de tener menos horas presénciales del alumno, lo que dificulta la adquisición de estas competencias en las clases. En este trabajo se presenta algunas reflexiones de un modelo de tutoría basada en la entrevista personal y reuniones en pequeños grupos. El objetivo de este trabajo es pues mostrar las ventajas del modelo de tutoría mencionado y estudiar sus posibilidades para afianzar algunas de las competencias transversales del Espacio Europeo de Educación Superior. Este modelo se ha visto útil en el desarrollo personal y humano del alumnado, y ha servido para detectar carencias desde el punto de vista personal, académico y curricular. Además el modelo de tutoría propuesto permitirá afianzar muchas de las competencias transversales personales, sistémicas e instrumentales que son parte de los nuevos títulos de grado. Se pretende que a través del modelo propuesto, se contribuya a que el alumnado sea artífice de su particular proyecto de vida y su propia cualificación, potenciar su capacidad de pensar y de hacer crítica, desarrollar su capacidad para planificar y llevarlo a la práctica.
\end{abstract}

Palabras clave: tutoría, títulos de grado, ingeniería agronómica, competencias transversales.

ABSTRACT: The new degrees incorporate generic skills, dedicated to training students in human values. In addition these new degrees have fewer hours in classroom for the students', this point makes it difficult to acquire these skills. This paper presents some reflections of a model of Tutoring based on personal interviews and meetings in small groups. The aim of this paper is therefore to show the mentioned advantages of tutoring model and consider its options to secure some of the generic skills of the European Higher Education Area. This model has been useful in students' personal and human development, and has served to identify lacks from several points of view: personal, academic and curriculum. In addition, the proposed tutoring model will consolidate many of the generic skills, which are part of the new degrees: personal, systemic and instrumental. It is intended that through the tutoring proposal, the students are the architect of their particular life plan and their own skills, enhance their ability to think critically and to develop its capacity to plan and put it into practice.

Key words: tutoring, university degree, agronomic engineering, skills and competences. 
San-Antonio-Gómez, C. \& Manzano Agugliaro, F. (2010). La tutoría como vehículo para el fomento de las competencias transversales en los nuevos grados de ingeniería agrónoma. Espiral. Cuadernos del Profesorado [en línea], 3(6), 73-83. Disponible en: http://www.cepcuevasolula.es/espiral.

Fecha de recepción: 29/05/2010

Fecha de aceptación: 17/08/2010
Enviar correspondencia a: fmanzano@ual.es

\section{1.- INTRODUCCIÓN.}

Si el Espacio Europeo de Educación Superior (EEES) busca formar estudiantes a través del aprendizaje a lo largo de la vida, de forma que sean ellos mismos quienes tomen las riendas de su carrera, evitando que su paso por la Universidad sea una mera absorción de conocimientos, y estructura la carrera en el trabajo y esfuerzo personal continuado realizado fuera de las aulas, a través de los créditos ECTS (European Credit Transfer System), se hace imprescindible contar con un tutor que asesore y marque al alumno el camino a seguir para poder completar su paso por la Universidad.

Como consecuencia, la tutoría se convierte en un elemento clave del EEES, en una herramienta fundamental para desarrollar la nueva metodología y alcanzar los objetivos propuestos por el mismo. Y esto es así por las siguientes razones (Ordóñez y Pérez, 2006; Martínez y Crespo, 2010):

- El EEES exige al alumno un mayor protagonismo en su devenir académico, lo que implica una atención más personalizada que sepa adaptarse a las especiales y personales circunstancias de cada alumno.

- La gran carga de trabajo que supone el nuevo sistema a través de los créditos ECTS, exige, igualmente, un asesoramiento más riguroso, y la elaboración de planes de estudio a la carta para los alumnos.

- El nuevo rol más activo del alumno, como auténtico protagonista de su entorno de aprendizaje, tiene como contraprestación para el profesorado nuevas acciones de apoyo personalizado que desarrollen sus capacidades.

Por lo tanto, las nuevas exigencias del EEES sitúan a la figura del tutor y su función tutorial como clave de desarrollo del mismo y, en definitiva, como indicador de calidad (Gairín et al., 2004) que, no olvidemos, es uno de los objetivos fundamentales de la Declaración de Bolonia. No obstante, creemos que la tutoría no debería limitarse a ser un mero indicador de calidad, sino que debe ir más allá, para convertirse en el revulsivo, en el elemento clave y dinamizador de la calidad en la enseñanza, pues sirve para detectar tanto los puntos fuertes y débiles del sistema educativo, las nuevas necesidades que surgen $\mathrm{y}$, sobre todo, las demandas reales de los alumnos.

En cuanto a la figura del tutor, hay que superar su vieja configuración para convertir al tutor no sólo en ese apoyo personalizado del alumno que le orienta en su itinerario curricular y académico, si no que también lo haga en su vida personal, y trata de obtener del mismo sus máximas habilidades y competencias que le hagan destacar para conseguir su éxito universitario. Es decir, la tutoría debe englobar diversas funciones y aunar en una sola figura los tradicionales tipos de tutoría (Lazaro, 1997; Boronat et al., 2005). Así, un buen tutor, conforme al nuevo sistema de educación superior debe desarrollar la tutoría académica, ceñida al ámbito científico; la docente, en la que la tutoría se convierte en una especial forma de docencia a través de grupos pequeños; así como la tutoría personal, que abarca el ámbito más privado del alumno (Meseguer et al., 2009).

Este modelo de tutor es el que proponemos y que desarrollaremos a continuación basándonos en la experiencia acumulada en los últimos años que llevamos aplicándolo y perfeccionando en la Escuela Técnica Superior de Ingenieros (E.T.S.I.) Agrónomos de la Universidad Politécnica de Madrid (UPM). Como antecedente partimos de sistema de tutorías implantado en la citada escuela en el curso 2005-2006, para después exponer el modelo con sus objetivos y metodología. Por último avanzamos cómo será la adaptación e implementación de dicho modelo de tutoría transversal a las necesidades del EEES, reuniendo un elenco de competencias transversales que, según los expertos, debería tener un ingeniero en la actualidad y que nos 
proponemos que adquieran los alumnos que participen en nuestro modelo tutorial. Se trata, por tanto, de que el alumno se implique en la consecución de competencias y habilidades que tienden a su capacitación profesional, pero especialmente, las tendentes a fomentar aspectos éticos, sicológicos, sociales o de relación personal y tolerancia, de liderazgo, de compromiso social o medioambientales.

El objetivo de este trabajo es, pues, mostrar las ventajas del modelo de tutoría mencionado y estudiar sus posibilidades para afianzar las competencias transversales del EEES en los nuevos títulos de grado en general, y en particular en los de ingeniería agronómica.

\section{2.- EL SISTEMA ACTUAL DE TUTORÍAS EN LA E.T.S.I. AGRÓNOMOS DE LA UPM.}

En el curso 2005-2006, se implantó en la E.T.S.I. Agrónomos de la UPM, un sistema de tutorías. Su objetivo era elevar la calidad educativa de los alumnos, procurar su integración en la Escuela y, mediante el consejo y la orientación del tutor, conseguir una adecuada formación conducente a la conclusión de su carrera con éxito, dentro de los plazos previstos. La implantación del sistema de tutoría se basaba en:

1. La futura integración del Sistema Universitario Español en el Espacio Europeo de Enseñanza Superior (EEES).

2. El derecho del alumno a "Disponer de un sistema de tutela que facilite el aprendizaje y la elección del currículum” (Estatutos de la UPM, Art. 123, apartado g).

3. El desconocimiento del mundo universitario y el funcionamiento de la Escuela de los alumnos de nuevo ingreso.

4. La demanda durante años de la Delegación de Alumnos.

El Sistema de Tutoría se implantó en dos etapas ( $1^{\circ}$ y $2^{\circ}$ ciclo) $y$, desde entonces, lo llevan a cabo docentes voluntarios. Como inicio el ICE (Instituto de Ciencias de la Educación) de la UPM, impartió a todos los tutores una jornada de formación sobre la Tutoría en la Universidad.

a) Tutorías para alumnos del $1^{\text {er }}$ ciclo: realizado fundamentalmente por docentes que imparten asignaturas básicas. Sus objetivos para con el alumno son:

- Su permanencia en la Escuela
- Asesorarle en aquellos aspectos que inciden en su rendimiento académico.

- Que progrese en sus estudios, evitando dejar asignaturas Troncales y Obligatorias

- Asesoramiento en la elección de materias optativas y de libre elección, así como la elección de orientación del $2^{\circ}$ ciclo.

- Ayuda respecto a otros problemas o iniciativas y en caso de no poder resolverlos personalmente, encauzarlos hacía otros docentes o instituciones de la Escuela o de la Universidad.

b) Sistema de Tutoría de $2^{\circ}$ ciclo: lo desarrollan principalmente docentes de la orientación que el alumno haya elegido. Su objetivo es orientar al alumno en su especialidad y asesorarle en las cuestiones antes reseñadas e informarle sobre Becas, Prácticas en Empresas, Cursos de Formación, salidas profesionales, etc.

La asignación del Tutor tanto para alumnos de $1^{\text {er }}$ ciclo como de $2^{\circ}$ ciclo, se realiza de forma aleatoria entre los docentes voluntarios. El ser Tutor de $1^{\text {er }}$ ciclo no implica que no se pueda ser de $2^{\circ}$ y viceversa y por lo tanto el que un alumno pueda ser tutorado por un solo Profesor Tutor a lo largo de toda la carrera.

Se aconseja que el Profesor Tutor mantenga reuniones periódicas con el alumno tutorado (al menos tres al año), la reunión inicial a principio de curso para establecer el primer contacto con el alumno y confeccionar la ficha de control y seguimiento, la segunda reunión sería en mayo, previa a los exámenes de junio, y la tercera en septiembre, después de los exámenes y antes de la matrícula del siguiente curso.

\section{3.- EL MODELO DE TUTORÍA TRANSVERSAL PUBLICADO. ACTUALMENTE}

El modelo que se presenta se diseñó para ser aplicado en el sistema tutorial de la E.T.S.I. Agrónomos de la UPM antes mencionado, aunque sus objetivos han sido más ambiciosos al querer desarrollar algunos de los paradigmas de Bolonia. En un principio, estaba dirigido a los alumnos del primer ciclo, pero muchos de ellos, cuando pasan al segundo, siguen acudiendo e estas tutorías.

La tutoría universitaria, según algunos autores, es la base fundamental de la educación 
académica y moral de Occidente (Gordon y Gordon, 1990). La tutoría, en sus inicios, suponía encargar a un docente la formación de un alumno en su totalidad, velando el tutor por el desarrollo de su personalidad y constituyéndose en garante de su grado de cientificidad y formación (Lázaro, 2004). Obviamente, esta ambiciosa concepción tenía mucho que ver con las limitaciones de las primeras universidades, pero nunca dejó de ser un ideal educativo ensalzado y desempeñado por tutores como Platón, Aristóteles, Locke o Rousseau (Levin, 1992).

Es en las universidades anglosajonas donde el modelo tutorial ha permanecido en el tiempo y es allí donde al principio acudimos para fundamentar nuestro sistema. Si nos remontamos a experiencias sobre lo que ha sido tutorial system, nos encontramos con la clásica definición de Moore que lo describe de manera sencilla: "reunión semanal del estudiante -solo o en pequeños grupos- de aproximadamente una hora de duración, con el tutor que le ha sido asignado" (More, 1968). También con la no menos clásica afirmación de Caldin: "es un tiempo de conversación individual y discusión en el que el alumno adopta una postura activa y no pasiva como en la clase teórica” (Caldin, 1968). En el tutorial, el docente se preocupa de desarrollar las capacidades del alumno y la materia se usa, fundamentalmente, como medio para promover ese desarrollo. Sin embargo, en nuestro caso, al plantearse una tutoría transversal, su objetivo no será desarrollar las capacidades del alumno en una materia concreta, sino desarrollar hábitos de trabajo, actitudes, capacidades generales que más adelante se expondrán.

El sistema es tanto más exitoso cuando se logra una relación de amistad y confianza que, desde el momento de su ingreso en la Escuela, se procura establecer con el estudiante; relación que continúa, en la mayoría de los casos, durante todo el período del segundo ciclo y, con frecuencia, también posteriormente.

La tutoría está orientada esencialmente a la formación individual del estudiante. Buena parte de los estudios sobre el tema, muchos de los cuales han sido ya citados en este trabajo, se refieren al modo en que esa tarea de formación individual puede realizarse con mayor eficacia. Pero la mejora del aprendizaje y la formación individual no es el único partido que puede obtenerse de una buena tutoría.

Además, es importante que el tutor tenga otras cualidades que, en la medida de lo posible, procuramos poner en práctica como: saber escuchar, discreción, ecuanimidad, objetividad, comprensión, sentido positivo, confiar en la palabra del alumno. En definitiva, no basta que el tutor domine y se centre solamente en los aspectos académicos y universitarios sino que debe conocer al alumno para buscar su desarrollo integral -incluido personal- de sus alumnos, respetando su originalidad e intimidad, ganándose su confianza para saber exigirle con suavidad y firmeza. Tal vez entonces, encuentre todo el eco que merece la tutoría y el asesoramiento académico especializado.

\section{1.- Objetivos.}

El objetivo fundamental es conseguir que el alumno desarrolle actitudes y capacidades y adquiera las competencias transversales $\mathrm{o}$ generales propias del estadio académico en que se encuentra, parejo a su progreso integral como persona. Podría concretarse en que el alumno se acostumbre a pensar por sí mismo -lo que no es habitual en la mayoría de los universitarios-, que se plantee problemas y sus posibles soluciones, que aprenda a organizar su tiempo y que constate que es un ser social y solidario, y no un simple individuo de la especie carente de responsabilidades. Podríamos decir también que se trata de ayudar al alumno a tomar decisiones libre y responsablemente tanto en lo académico y profesional, como en lo personal. Estos objetivos también podrían concretarse:

- Desarrollar al máximo la personalidad del alumno

- Conseguir que se oriente a sí mismo

- Que se comprenda y acepte

- Que madure para tomar decisiones

- Que se adapte al medio

- Que aprenda más y mejor

- Que aprenda a pensar (Rodríguez, 2002)

Los alumnos hasta ahora tutorados, reconocen sus carencias en lo personal:

- Tienen problemas para hacer uso de su libertad. Carecen de experiencia.

- Falta de seguridad en sí mismos. Se manifiesta, por ejemplo, en no preguntar en clase.

- No se conocen a sí mismos, ni sus capacidades ni sus limitaciones.

- Estado de ánimo muy variable: o se sobrevaloran o se infravaloran.

- Dejan las cosas para después. 
Los alumnos hasta ahora tutorados, reconocen sus carencias en lo académico:

- Desconocen soluciones para superar sus fracasos.

- Les falta método de estudio. Tienen dificultades con las técnicas de trabajo intelectual.

- Les cuesta integrar conocimientos.

- No saben organizar su tiempo, se podría incluso decir, sin temor a exagerar, que tienen alergia a hacerse un horario y cumplirlo.

Los alumnos hasta ahora tutorados, reconocen sus carencias lo curricular:

- No siempre saben decidir en la selección de asignaturas, de especialidades, de asignaturas que dejan para septiembre.

\section{2.- Metodología.}

Como se ha dicho antes, el sistema tutorial que proponemos está dirigido a los alumnos del primer ciclo que son a los que en principio nos corresponde atender, pero muchos de ellos, cuando pasan al segundo, siguen acudiendo a las tutorías, por lo que se ha diseñado un módulo especial para ellos.

Las reuniones con los tutorados son de dos tipos: entrevista individual y reunión por grupos: alumnos de nuevo acceso, alumnos del primer ciclo y alumnos del segundo. Las reuniones con el alumno no se limitan a las tres que recomienda la Escuela, suelen tener una periodicidad mensual o quincenal según los casos. La relación entre el tutor y el alumno se procura que sea fluida: el alumno puede dirigirse al tutor en cualquier momento y solicitar orientación tanto para su labor académica como, en general, para su vida universitaria. Un rasgo común del método ha sido la informalidad (More, 1968), que responde, sobre todo, al hecho de que los encuentros no previstos constituyen una gran ayuda para la labor discente del tutor. Caldin (1968) expresa la misma idea cuando señala, como rasgo particular, la flexibilidad: en definitiva, se trata de una atención individualizada. La reunión con los alumnos habitualmente tiene lugar en el despacho del tutor.

La forma de conducir la reunión personal con cada alumno tutorado, varía en función de las características personales pero fundamentalmente se trata de que el tutor anime de forma constructiva, tratando de conseguir que el alumno de lo mejor de sí mismo tanto en lo académico como en lo personal. Para ello, es importante ganarse la confianza del alumno para conseguir que exprese, del modo más completo posible, sus dudas, problemas o dificultades con el estudio o todas aquellas circunstancias personales que lo afectan negativamente. Se trata además de que el alumno mejore un poco en cada sesión aunque, a veces, experimente un retroceso momentáneo.

En cuanto a las reuniones por grupos, hay variedad de maneras para conducirlas. Si seguimos acudiendo a la literatura científica tradicional para acercarnos al concepto británico de tutorial, vemos que depende, en primer lugar, del número de asistentes a cada sesión. Para algunos, no debería ser superior a seis y posiblemente dos es el mejor número (Caldin, 1968). MacKenzie, clasificando los grupos por su tamaño entiende como tutorial solamente a los grupos de 1 a 3 alumnos (Mackenzie et al., 1970). Moore, analizando la práctica de este método en Oxford en los años setenta del pasado siglo, afirma también que pueden estar presentes dos, o incluso tres alumnos; pero centra su análisis en el sigle-tutorial, tutorial para un solo alumno (More, 1968). En definitiva, el número ideal de alumnos que en esa literatura científica tradicional propone, parece ser el de uno o dos; desde luego, no debería sobrepasar nunca los cuatro alumnos. En nuestro caso, las reuniones con los alumnos de nuevo acceso son para el grupo de 2 a 4 que nos ha sido adjudicado por el Coordinador Docente y de Calidad. Para los siguientes cursos del primer ciclo (tanto de primero como de segundo curso) el número de alumnos varía entre 4 y 10 . Las reuniones para alumnos del segundo ciclo, al ser totalmente voluntarias varían entre 8 y 15 .

En estas reuniones, el tutor expone un tema previamente anunciado en la reunión anterior, normalmente elegido por él, aunque para los grupos del segundo ciclo, se desarrollan temas que sugieren los propios alumnos. La exposición suele hacerse con PowerPoint o como lección magistral duración 20-30 minutos, dejando un periodo de discusión de unos 15 minutos. Se suele recomendar bibliografía fácil de conseguir e, incluso, se suministran fotocopiados resúmenes de lo tratado. Como los temas suelen seguir un orden previamente establecido, al principio de cada tema hace un recordatorio del anterior que sirve de introducción del nuevo.

La forma de conducir ese proceso, varía en función de los temas tratados -que suelen ser sobre la formación en valores- y de las 
características personales, pero básicamente se trata de conseguir que el alumno piense y, sobre todo, que profundice en las ideas o tesis contenidas en la exposición. Lo importante no es tanto la crítica -en el sentido de juzgar algo con arreglo a ciertas normas preestablecidas-, sino más bien hacer que el alumno, después de reflexionar sobre el tema, se forme su propio juicio. En otras palabras, ayudar a los alumnos a que "construyan su propio conocimiento" (Bain, 2007) y, en consecuencia, como el obrar sigue al ser, labren su personal comportamiento de forma responsable. Todo ello según la idea de que los estudiantes "deben aprender los hechos a la vez que aprenden a utilizarlos para tomar decisiones sobre lo que entienden y lo que no" (Bain, 2007). En cualquier caso, Moore sostiene que el tutorial es un método de búsqueda, de prueba, concluyendo que, con él, se prefiere lo que es un intento a lo definitivo, el ensayo al resultado. Es decir, se concede mayor importancia al porqué (actitud crítica) que al qué (contenido objetivo) (Bain, 2007).

En el trasfondo de las posibles variaciones, debidas tanto al número de alumnos como a la diversidad de los temas tratados en las reuniones y a la informalidad peculiar del sistema, el planteamiento del proceso de enseñanzaaprendizaje en el tutorial reviste dos características principales: la participación activa del alumno -con el trabajo personal que implicay el pensamiento crítico, consecuencia directa de la conversación sostenida entre alumno y tutor. Estas características obedecen a una concreta teoría del aprendizaje y a una particular actitud ante el conocimiento. Aunque estas bases sean susceptibles de una valoración más detallada, se puede anotar la importancia que este método reconoce al trabajo personal y a la actividad del alumno, así como al análisis del conocimiento adquirido y revisado críticamente (Highet, 1954).

\section{3.- Alumnado del primer ciclo.}

La labor fundamental del tutor del primer curso es el seguimiento del proceso de adaptación del alumno a la vida universitaria, acompañándole en la transición de los estudios secundarios a los universitarios (Salaburu, 2007). Una de las primeras metas a conseguir es que el alumno conozca a nuevos compañeros y que tenga a su lado al tutor al que puede acudir para resolver las numerosas dudas que le surgen los primeros días a cerca del funcionamiento y organización del curso. Para ello el tutor organiza inmediatamente después de habérsele comunicado quienes son sus tutorados, una primera reunión convocada por email o por teléfono, para que todos -tutor y alumnos- se conozcan entre sí. Además esta primera reunión del grupo ayuda a vencer la timidez propia de su edad, y sirve para aclimatar y comenzar a acortar la lógica distancia tutoralumno que, con el tiempo, acabará en confianza mutua y, en muchos casos, amistad duradera. En ésta reunión se les explica en qué consiste la tutoría y se les cita a una primera entrevista personal en esa misma semana.

\section{Entrevista personal.}

En la primera entrevista con el alumno, se hace una toma de contacto en la que éste cumplimenta una ficha que proporciona la Escuela, con sus datos personales y académicos, y se le anima a que manifieste cuales son los problemas o dificultades de todo orden que ha encontrado en sus primeros días. A partir de este momento se genera el marco adecuado para dar comienzo al asesoramiento personalizado dirigido a que el tutorando obtenga el máximo aprovechamiento en lo académico y en lo personal.

En la siguiente entrevista, unos días más tarde, el tutor le ayuda a planificar cada día de la semana organizando su tiempo de trabajo personal, tanto en los días lectivos como en los fines de semana. Para ello el alumno con la ayuda del tutor rellena un modelo de horario a modo de tabla de 8 de la mañana a 10 de la noche para los 7 días de la semana, en el que figuran las horas dedicadas a sus actividades académicas grupales (clases, prácticas, trabajos en equipo, etc.) y las que teóricamente puede dedicar al trabajo personal. En la última fila de la tabla, y para cada día de la semana, se reflejan estas horas.

Una característica común de los alumnos de los primeros cursos es su propensión al autoengaño en lo referente al tiempo que dedican al estudio, y la tendencia a dejar las cosas para otro momento. Para ello se les anima a que contabilicen las horas dedicadas al estudio personal y así objetivar ese tiempo, de la misma manera que, por ejemplo, también se mide la temperatura corporal. Ese control lo pueden hacer con la Tabla 1. En la primera columna figuran las horas de estudio que pueden contabilizarse enteras, medias e, incluso, cuartos de hora. En la antepenúltima fila, figuran los 31 días del mes; en la penúltima, el día de la semana que corresponde a cada cifra en ese mes (en la tabla se pone el ejemplo real de una semana de abril de 2009, de un buen alumno de primer curso que 


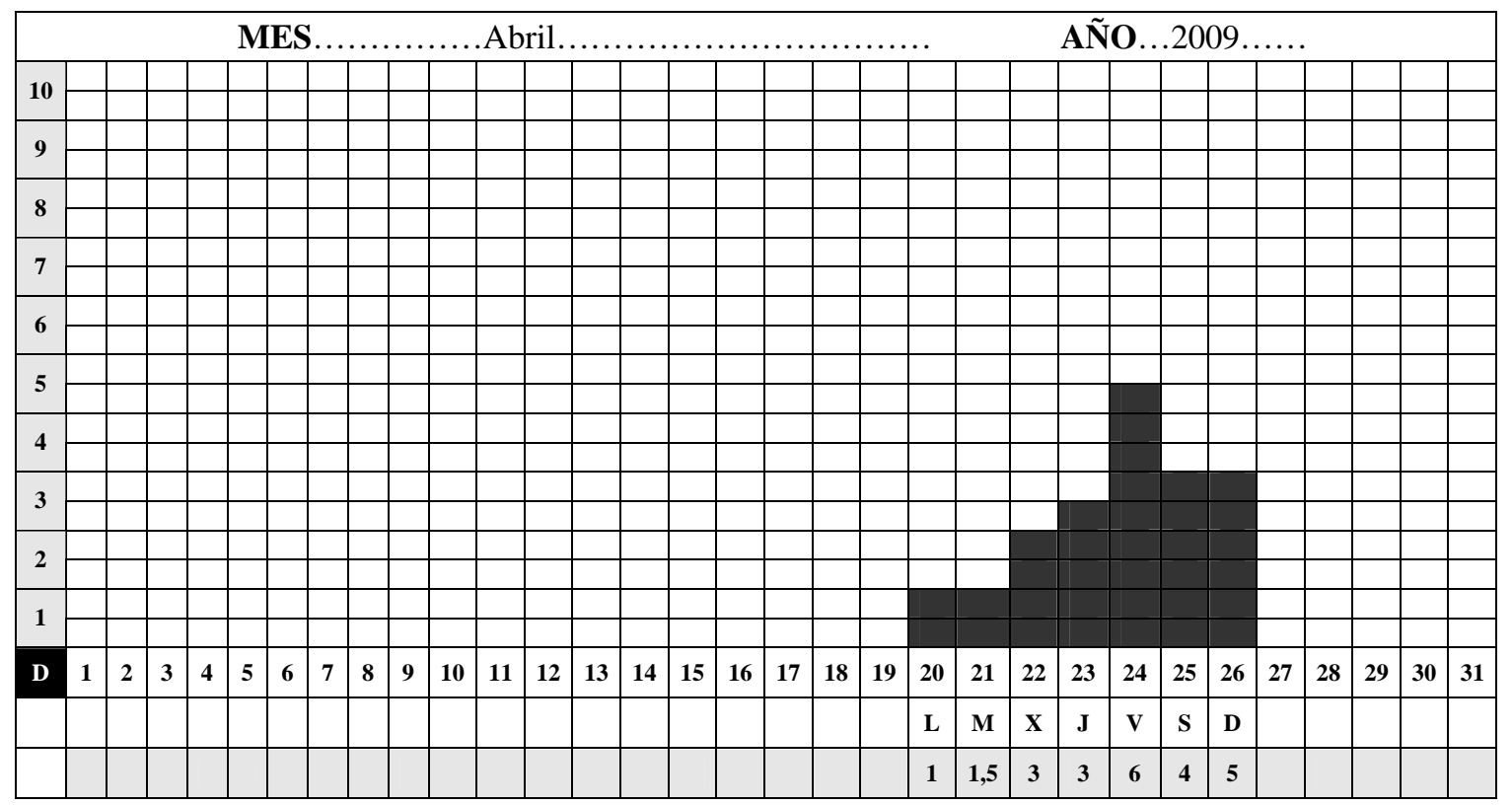

Tabla 1.- Control mensual del estudio personal (ejemplo una semana de abril de 2009)

aprobó todas las asignaturas del primer cuatrimestre); en la última fila, las horas teóricamente posibles que ese alumno puede estudiar en cada día de la semana. Al rellenar los datos reales de estudio, se comprueba que dicho alumno solamente el lunes logró los objetivos de estudio previstos para cada día de la semana. En la gráfica se advierte, que en el plan de estudios actual, el alumno dispone de muy poco tiempo para dedicarlo al estudio personal. El mayor número de horas posibles corresponden al fin de semana en el que, lógicamente, tiene que descansar.

En las sucesivas entrevistas se chequean las dos tablas para ver los ajustes de horario y las modificaciones que sean pertinentes. El tutor conserva una copia de cada hoja de estudio de cada mes con el fin de valorar los distintos diagramas de barra que vayan saliendo y buscar con el alumno, si es el caso, las posibles razones por lo que no se han cumplido los objetivos. En esas entrevistas se recomienda al alumno que cuide las horas de sueño -hemos observado que generalmente no duermen lo suficiente lo cual influye negativamente en su atención en clase y, en general, en su rendimiento académico- y que dedique un tiempo semanal al deporte.

\section{Reunión de grupo.}

Como se ha dicho, en estas reuniones el tutor expone un tema previamente anunciado durante 20-30 minutos, seguido de un periodo de discusión de otros 15 minutos. Los temas tratados van dirigidos al desarrollo de las competencias transversales, es decir su formación en valores humanos con el objetivo de conseguir que el alumno piense $\mathrm{y}$, sobre todo, que profundice en las ideas contenidas en la exposición para que después de reflexionar sobre el tema, se forme su propio juicio $\mathrm{y}$, por consiguiente, aprendan a asumir responsabilidades. En los tres primeros años del primer ciclo los temas tratados son:

1. El conocimiento propio

2. La aceptación de sí mismo

3. El orden: La organización y gestión del tiempo

4. La motivación intrínseca hacia el estudio

5. Las causas del desánimo y la pereza

6. La superación personal

7. El dominio de sí mismo

8. El hábito de pensar

9. El hábito de leer

10. Los valores como cualidades del hombre

11. Humildad

12. Sencillez. Sinceridad

13. Alegría. Optimismo, sentido positivo de la vida

14. Los valores de la convivencia

15. Comprender, tolerar, respetar y disculpar

16. La amistad y el compañerismo

17. La búsqueda de la verdad

18. Razón y sentimiento

19. Libertad y responsabilidad 


\section{El compromiso social: voluntariado}

\section{4.- Alumnado del segundo ciclo.}

\section{Entrevista personal.}

La clave de la tutoría que se realiza durante el segundo ciclo, es que se concibe en sentido amplio, pero que se ocupa de aspectos concretos y relevantes para el estudiante. De un lado, a través del asesoramiento del currículo académico propio del plan de estudios. De otro, mejorando sus hábitos de estudio que comprenden la planificación y organización de horarios, técnicas de estudio, algo a lo que ya están acostumbrados por su experiencia anterior. También en este ciclo se recomienda al alumno elaborar su modelo de horario y su control mensual de horas de estudio (Tabla 1). Hemos verificado, también para estos alumnos, la validez de ambas tablas y su utilidad para planificar su trabajo. También se les sugiere la conveniencia de tener experiencias internacionales como el programa Erasmus, participar en congresos de estudiantes, prácticas en empresas, actividades de voluntariado, etc., en definitiva, todo aquello que afiance su formación integral. En este periodo se fomenta igualmente la importancia de tener un buen expediente académico y un alto nivel de idiomas. Una clave de la tutoría personalizada es que requiere un perfil para el alumno medio y otro para los que destacan especialmente, potenciando la excelencia académica del alumno a través de la petición de becas, y su implicación en los departamentos de la Escuela más acordes con sus capacidades personales, participando, por ejemplo, en proyectos de investigación que allí se desarrollen.

\section{Reunión de grupo}

Además de los aspectos académicos y personales, interesa potenciar especialmente lo relacionado con las capacidades transversales del ámbito profesional. En efecto, en este periodo, se fomenta con más ímpetu el espíritu crítico de los estudiantes, la capacidad de análisis y síntesis, la capacidad de organización y planificación, la responsabilidad ética y el compromiso social, etc. A ello van dirigidas las reuniones de este segundo ciclo que son más esporádicas y están más sujetas a peticiones concretas de los tutorados. De hecho, se han dado sesiones con invitados que desarrollen temas específicos. Los temas fijos que se tratan son:

1. El respeto al medio ambiente.

2. La gestión del éxito y del fracaso.
3. Cuestiones éticas del trabajo profesional.

4. Diversidad y multiculturalidad.

5. Responsabilidad social corporativa.

\section{4.- ADAPTACIÓN DEL MODELO AC- TUAL DE TUTORÍA TRANSVERSAL A LAS NECESIDADES DEL EEES.}

Como dijimos en el punto anterior, el modelo tutorial que aplicamos está en concordancia con el que se pretende implantar en el EEES. La adaptación solamente afecta a la redefinición en su redacción más que en el contenido, de las competencias generales o transversales y en su ajuste a la nueva tipología del grado en lugar de los antiguos primer y segundo ciclo de la carrera. El objetivo fundamental no cambia, sigue siendo el conseguir que el alumno desarrolle actitudes y capacidades y adquiera las competencias transversales o generales propias del estadio académico en que se encuentra, es decir, procurar su progreso integral como persona. En este sentido, las intenciones y metas de nuestro modelo de tutoría se orientan hacia uno de los cuatro pilares que la Comisión de Jacques Delors señaló: el saber ser (los otros tres son: saber, saber hacer y saber estar). Evidentemente el saber ser, tiene que ver con el saber cómo ser, es decir los valores como forma integrante de percibir a los otros y vivir en un determinado contexto sociocultural. Podríamos decir también, que el saber ser tiene que ver con el desarrollo integral personal que implica, entre otras cosas, lo que hasta ahora hemos venido procurando: que el alumno aprenda a pensar por sí mismo y se forme su propio criterio para tomar decisiones libre y responsablemente, tanto en lo académico y profesional, como en lo personal. Para ello, es necesario que se plantee problemas y sus posibles soluciones, que aprenda a organizar su tiempo y que constate que es un ser social y solidario con responsabilidades tanto personales como sociales.

\section{1.- Objetivos.}

En el Documento-Marco sobre la integración del Sistema Universitario Español en el EEES (Ministerio de Educación, Cultura y Deporte, 2003), se afirma: "Los objetivos formativos de las enseñanzas oficiales de nivel de grado tendrán, con carácter general, una orientación profesional, es decir, deberán proporcionar una formación universitaria en la que se integren armónicamente las competencias generales básicas, las competencias transversales 
relacionadas con la formación integral de las personas y las competencias más específicas que posibiliten una orientación profesional que permitan a los titulados una integración en el mercado de trabajo". Como ha quedado dicho, el objetivo que nos proponemos es, precisamente, trabajar en los tutorados las competencias transversales relacionadas con la formación integral de las personas.

Se hace necesario definir cuales son estas competencias transversales que debería tener un ingeniero en la actualidad y que tendría que ir adquiriendo cada alumno de ingeniería:

- Una respuesta podría ser: poseer un profundo conocimiento de las cuestiones éticas, ser tolerante, cultivado en humanidades y estar dispuesto a aprender y a asumir responsabilidades (Hedberg, 2001).

- Otra respuesta la encontramos en los requisitos que plantea ABET (Accreditation Board for Engineering and Technology) en sus "Engineering criteria 2000" (Lattuca et al., 2006) donde se estipula que los programas de ingeniería deben demostrar que sus graduados tienen comprensión de las responsabilidades éticas y profesionales (an ability to understand professional, ethical and social responsibilities).

- En la Thematic Network E4 (Enhancing Engineering Education in Europe), para todas las ingenierías se dice: mostrar un buen conocimiento de la influencia de la actividad del ingeniero en los distintos aspectos de la vida y el medio ambiente y demostrar un acercamiento altamente ético y moral a las tareas de la ingeniería (display an understanding of the influence of engineering activity on all life an the environment, and demonstrate a high moral and ethical approach to engineering tasks).

- En el proyecto Tuning (Tuning Project II, Mayo 2004), entre las competencias transversales o genéricas que se señalan tenemos:

- Competencias instrumentales, como la planificación y gestión del tiempo (planning and time management), $\mathrm{y}$ la toma de decisiones (decisionmaking).

- Interpersonales, como el compromiso ético (ethical commitment); la capa- cidad crítica y autocrítica (critical and self-critical abilities); habilidades interpersonales (interpersonal skills); y el reconocimiento de la diversidad y multiculturalidad (appreciation of Diversity and multiculturality)

- En el Libro Blanco de la ANECA, se señalan las del proyecto Tuning antes citado:

- Competencias instrumentales, como la capacidad de organización y planificación; y la toma de decisiones.

- Personales: compromiso ético; razonamiento crítico; habilidades en las relaciones interpersonales; reconocimiento de la diversidad y multiculturalidad.

- Las competencias transversales de la Universidad de Almería para los títulos de grado:

1. Conocimientos básicos de la profesión

2. Habilidad en el uso de las TIC (Tecnologías de la Información y el Conocimiento)

3. Capacidad para resolver problemas

4. Comunicación oral y escrita en la propia lengua

5. Capacidad de crítica y autocrítica

6. Trabajo en equipo

7. Conocimiento de una segunda lengua

8. Compromiso ético

9. Capacidad para aprender a trabajar de forma autónoma

10. Competencia social y ciudadanía global

En los nuevos títulos de grado las Competencias transversales se clasifican en tres bloques:

- Instrumentales: Capacidad de análisis y síntesis, Capacidad de organización y planificación, Comunicación oral y escrita, Conocimiento de lengua extranjera, Conocimiento de informática, Capacidad de gestión de la información, Resolución de problemas, y Toma de decisiones.

- Personales o Interpersonales: Trabajo en equipo, Trabajo en un contexto 
internacional, Habilidades en las relaciones interpersonales, Reconocimiento de la diversidad y multiculturalidad, Razonamiento crítico y Compromiso ético.

- Sistémicas: Creatividad, Liderazgo, Aprendizaje autónomo, Adaptación a nuevas situaciones, Conocimiento de otras culturas y costumbres, Iniciativa y espíritu emprendedor, Motivación por la calidad, y Sensibilidad por temas medioambientales.

Pensamos que los listados anteriores, además de repetitivos, no abarcan la complejidad de la formación personal integral. A nuestro juicio habría que añadir las siguientes competencias transversales en el ámbito de lo personal:

- Construir una escala de valores personal.

- Conocimiento de sí mismo.

- Motivación por el estudio.

- Disciplina personal: no huir del esfuerzo. Sentido positivo del esfuerzo. Aprender a trabajar cansado.

- Autorrealización.

- Gestión del éxito y del fracaso.

\section{2.- Metodología.}

La metodología que se propone aplicar en los nuevos títulos de grado no cambiará en lo sustancial con la descrita anteriormente y nos remitimos a ello. Las reuniones con los tutorados serán, igualmente, la entrevista individual, con una periodicidad mensual o quincenal y la reunión por grupos. En cualquier caso, tanto en las entrevistas como en las reuniones, se trata de que cada alumno tutorado se supere a sí mismo tanto en lo académico como en lo personal para lo cual habrá que ayudarle a vencer sus posibles dificultades con el estudio o todas aquellas circunstancias personales que lo afectan negativamente. En este sentido, consideramos que los temas tratados en las reuniones por grupos expuestos anteriormente, siguen siendo válidos porque son los más adecuados a las competencias transversales o genéricas que pretendemos que adquiera el alumnado, tanto en las personales, sistémicas o instrumentales.

\section{5.- CONCLUSIONES.}

En el presente trabajo se destaca la importancia de la tutoría dentro de la enseñanza universitaria, se pone de relevancia su importancia dentro de los nuevos títulos de grado adaptados al Espacio Europeo de Educación Superior.

Se ha planteado un modelo de tutoría con dos vertientes: entrevista personal y reuniones en pequeños grupos. Este modelo se ha visto útil en el desarrollo personal y humano del alumnado. Ha demostrado su utilidad para detectar por el docente y autodetectar por el discente carencias desde el punto de vista personal, académico y curricular. Además el modelo de tutoría propuesto permitirá afianzar muchas de las competencias transversales personales, sistémicas e instrumentales que son parte de los nuevos títulos de grado.

Se pretende que mediante la tutoría propuesta, contribuir a que el alumnado tutorado sea artífice de su particular proyecto de vida y su propia cualificación, que aprenda a organizar su tiempo y que constate que es un ser social y solidario con responsabilidades tanto personales como sociales. Esto supone: Potenciar la capacidad de pensar y de hacer crítica, desarrollar la capacidad para planificar y llevarlo a la práctica con una motivación y una escala de valores personal, todo ello compatible con una disciplina que le permita la autorrealización.

\section{6.- REFERENCIAS BIBLIOGRÁFICAS.}

Bain, K. (2007). Lo que hacen los mejores profesores de Universidad. Valencia: Publicaciones de la Universidad de Valencia.

Boronat Mundana, J., Castaño Pombo, N., \& Ruiz Ruiz, E. (2005). La docencia y la tutoría en el nuevo marco universitario. Revista Electrónica Interuniversitaria de Formación del Profesorado [en línea] 20, Vol. 8 (5), 69-74. Diponible en: http://www.aufop.com/aufop/uploaded_files/artic ulos/1247169241.pdf [Consulta: 2010, 14 de mayo].

Caldin, E.F. (1968). The Tutorial, en D. Layton (ed.), University Teaching in transition (58-62). Edinburgh: Oliver and Boyd Ltd.

Gairín, J., Feixas, M., Guillamón, C. \& Quinquer, D. (2004). La tutoría académica en el escenario de la educación superior. Revista Interuniversitaria de Formación del Profesorado [en línea] 18(1) 61$77 . \quad$ Disponible en: http://redalyc.uaemex.mx/pdf/274/27418105.pdf [Consulta: 2010, 20 de mayo].

Gordon, E.E. \& Gordon E. H. (1990). Centuries of tutoring. A history of alternative education in America and Western Europe. Lanham: University Press of America.

Hedberg, T., (2001). The role of the Global EngineerAn european view. Educating the Engineer for 
the 21st Century. Proceedings of the 3rd Workshop on Global Engineering Education. Kluwer Academic Publishers.

Highet, G. (1954). The Art of Teaching. New York: Vintage Books.

Lattuca, L. R., Terenzini, P.T. \& Volkwein, J. F. (2006). Engineering change: A study of the impact of EC2000. Ed. ABET (Accreditation Board for Engineering and Technology). Disponible en:

http://www.abet.org/Linked\%20DocumentsUPDATE/White\%20Papers/Engineering\%20Cha nge.pdf

Lázaro Martínez, A. (2004). Características y propuestas de la función tutorial en la universidad. En Blázquez Entonado, F.; Maynar Mariño, J. I. \&y Montanero Fernández, M. Materiales para la enseñanza universitaria: La formación de los profesores noveles universitarios. (189-212). Universidad de Extremadura, ICE.

Lázaro Martínez, A. (1997). La acción tutorial de la función docente universitaria. Revista Complutense de Educación 8(1) 233-252.

Levin, R.A. (1992). Review of "Centuries of tutoring. A history of alternative education in America and Western Europe". History of Education Quarterly, 32(3) 379-381.

Mackenzie, N., Eraut, M., \& Jones, H. C., (1970). Teaching and Learning. An Introduction to New Methods and Resources in Higher Education. Paris: UNESCO-IAU.

Martínez Lirola, M. \& Crespo Fernández, E. (2009). La tutoría universitaria en el modelo de la convergencia europea. En Gómez Lucas C. \& Grau Company S. Propuestas de diseño, desarrollo $e$ innovaciones curriculares $y$ metodología en el EEES (451-466). [en línea], Disponible

en: http://rua.ua.es/dspace/bitstream/10045/13199/23 /PROPUESTAS\%20CAP.\%2023.pdf [Consulta: 2010, 15 de junio].

Meseguer Velasco, S., Goñi Rodríguez de Almeida, M., Díez Estella, F., \& Hernando Masdeu J. (2009). La tutoría como elemento clave de la renovación permanente de la calidad universitaria. En f. Díez Estella, Actas del II Congreso de Innovación Docente en Ciencias Jurídicas (50).

Ministerio de Educación, Cultura y Deporte. (2003). La integración del sistema universitario español en el Espacio Europeo de Educación Superior. Documento-Marco Madrid: Ministerio de Educación, Cultura y Deporte [en línea] http://www.eees.es/es/documentacion [Consulta: 2010, 15 de junio].
Moore, W.G. (1968). The Tutorial System and its Future. Oxford: Pergamon Press.

Ordóñez Solana, C. \& Pérez López, Ma C. (2006). Orientación y tutoría en la Convergencia Europea: una experiencia en la Universidad de Granada. V Congreso internacional "Educación y Sociedad” [en línea] [Consulta: 2010, 15 de junio].

Rodríguez Moreno, M. L. (2002). Hacia una nueva orientación universitaria. Barcelona: Universitat de Barcelona, S.L. Unipersonal.

Salaburu, P. (2007). La Universidad en la encrucijada: Europa y EE.UU. Madrid: Academia Europea de Ciencias y Artes.

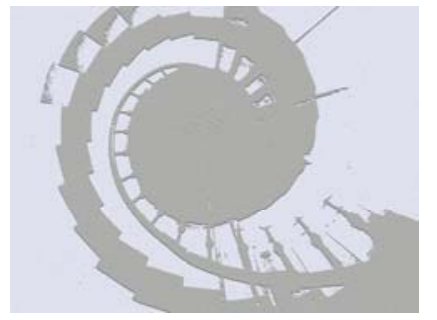

